\title{
PANCREATITIS BILIAR RECURRENTE ASOCIADA A VESÍCULA BILIAR DE LOCALIZACIÓN ANÓMALA Y COMUNICACIÓN INTERAURICULAR
}

\author{
RECURRENT BILIARY PANCREATITIS ASSOCIATED WITH ANALYZED BILIAR LOCALIZATION VESICLE \\ AND INTERAURICULAR COMMUNICATION \\ Alonso Soto 1,2,a, Manuel Ismael Ascarza-Huando 2,b
}

\begin{abstract}
RESUMEN
Mujer de 32 años que ingresa al servicio de medicina con cuadro clínico de pancreatitis biliar recurrente y colelitiasis sintomática. La ecografía abdominal y la colangioresonancia mostraron vesícula biliar en posición no habitual (posterior) y anomalía del árbol biliar. Incidentalmente se evidencia la presencia de una comunicación interauricular asociada a signos compatibles con hipertensión pulmonar. No hemos encontrado en la literatura asociación entre la presencia de esta anormalidad de la vesícula biliar y la presencia de comunicación auricular u otras cardiopatías congénitas.
\end{abstract}

Palabras clave: Pancreatitis recurrente; Vesícula biliar; Cardiopatía congénita. (fuente: DeCS BIREME)

\begin{abstract}
A 32-year-old woman admitted to the medical service with a clinical picture of recurrent biliary pancreatitis and symptomatic cholelithiasis. Abdominal ultrasound and cholangioresonance showed a gallbladder in an unusual position (posterior) and an anomaly of the biliary tree. Incidentally, the presence of an atrial septal defect associated with signs compatible with pulmonary hypertension is evident. We have not found in the literature an association between the presence of this gallbladder abnormality and the presence of atrial or other congenital heart disease.
\end{abstract}

Key words: Recurrent pancreatitis; Gallbladder; Congenital heart disease. (source: MeSH NLM)

\section{INTRODUCCIÓN}

La pancreatitis aguda es una patología relativamente frecuente con un significativa morbilidad y mortalidad. El $20 \%$ de las pancreatitis agudas son graves, y si la causa de la pancreatitis no se corrige, un $30-50 \%$ de los pacientes presentan recurrencias.

La pancreatitis aguda recurrente (PAR) es una entidad relativamente frecuente y potencialmente grave que requiere determinar la etiología y el tratamiento'.EI estudio etiológico con frecuencia necesita utilizar técnicas invasivas y costosas, algunas de las cuales tienen riesgo de complicaciones, incluyendo la misma pancreatitis ${ }^{2}$.
Cualquier causa que produce un episodio de pancreatitis aguda, puede potencialmente producir una recurrencia, especialmente si no se realiza el tratamiento adecuado. En un $7 \%$ de los pacientes coexisten varias etiologías, como por ejemplo la predisposición genética con otro factor obstructivo o tòxico.

La etiología de la PAR ha sido investigada en numerosos estudios, siendo el alcohol y la litiasis biliar las etiologías más prevalentes, englobando un $70 \%$ de los casos $^{4}$.

La pancreatitis aguda biliar puede ser una causa de PAR cuándo esta no se reconoce en un primer

'Departamento de Medicina, Hospital Nacional Hipólito Unanue, Lima-Perú.

${ }^{2}$ Instituto de Investigación en Ciencias Biomédicas (INICIB). Facultad de Medicina. Universidad Ricardo Palma, Lima-Perú.

a Médico Internista.

${ }^{b}$ Medicina de emergencias y desastres.

Citar como: Alonso Soto, Manuel Ismael Ascarza-Huando. Pancreatitis biliar recurrente asociada a vesícula biliar de localización anómala y comunicación interauricular. Rev. Fac. Med. Hum. Julio 2019; 19(3):101-105. DOI 10.25176/RFMH.v19i3.2154

Journal home page: http://revistas.urp.edu.pe/index.php/RFMH

Artículo publicado por la Revista de la Facultad de Medicina Humana de la Universidad Ricardo Palma. Es un artículo de acceso abierto, distribuído bajo los términos de la Licencia Creative Commons: Creative Commons Attribution 4.0 International, CC BY 4.0 (https://creativecommons.org/licenses/by/4.0/), que permite el uso no comercial, distribución y reproducción en cualquier medio, siempre que la obra original sea debidamente citada. Para uso comercial, por favor póngase en contacto con revista.medicina@urp.pe 
episodio, ya que la sensibilidad de la ecografía abdominal para detectar litiasis disminuye durante el episodio agudo, y se recomienda repetirla una vez resuelta. La resonancia magnética (CPRM) puede ser útil para detectar litiasis en las vías biliares, aunque su sensibilidad es menor para detectar las de pequeño tamaño ${ }^{5}$.Diversos fármacos están implicados en la etiología de la pancreatitis aguda recurrente. La hipertrigliceridemia con niveles superiores a 1000 $\mathrm{mg} / \mathrm{dL}$ puede ser una causa de PAR ya sea de causa hereditaria o alcoholica ${ }^{5}$. Asimismo, se ha descrito el tabaco como un factor independiente de PAR ${ }^{6}$.

Los factores genéticos pueden tener un papel, en la causa, la evolución y la recurrencia de la pancreatitis. Los genes implicados son el gen del tripsinógeno catiónico (PRSS1), el gen inhibidor de la proteasa serina Kazal tipo 1 (SPINK1), el gen regulador de la conductancia transmembrana de la fibrosis quística (CFTR) estando presentes en el $50 \%$ de la pancreatitis crónicas idiopáticas ${ }^{6}$. La enfermedad celiaca es una causa poco frecuente de PAR asociada a inflamación y obstrucción de la papila7. La pancreatitis autoinmune es una enfermedad fibroinflamatoria sistémica que pude afectar al páncreas ${ }^{8}$.El páncreas divisum es una frecuente anomalía congénita, con un prevalencia del 7-14\% ${ }^{9}$. La disfunción del esfínter de Oddi como causa de una PAR es todavía controvertida. Las neoplasias pancreáticas o ampulares pueden ser una causa de PAR por obstrucción del conducto pancreático. Los tumores neuroendocrinos, las neoplasias quísticas, la neoplasia mucinosa papilar intraductal (NPMI), y el adenocarcinoma pancreático pueden ser la causa de pancreatitis en alrededor del $2 \%$ de los pacientes s0-11. $^{10}$.

Las alteraciones o variedades anatómicas congénitas de la vía biliar extrahepática tienen gran relevancia desde el punto de vista quirúrgico, las variantes anatómicas observadas, tales como: vesícula biliar múltiple, plegada, multiseptal, y agenesia vesicular, localización no habitual; conductos biliares aberrantes, conducto cístico doble, o de gran longitud; y quistes de colédoco, entre los más frecuentes ${ }^{12}$. Se presenta el caso de una paciente con vesícula biliar de localización poco habitual, con anomalia del arbol biliar asociadas a pancreatitis aguda biliar recurrente y comunicación interauricular.

\section{REPORTE DE CASO}

Paciente mujer de 32 años, natural de Junín, procedente de Lima, ocupación ama de casa, conviviente, con antecedentes de litiasis vesicular y pancreatitis aguda en dos oportunidades 4 y 3 meses antes de su ingreso respectivamente. Inicia episodio actual hace 12 horas de manera insidiosa y curso progresivo caracterizado por dolor en epigastrio de irradiación lumbar, de moderada intensidad y asociado a náuseas y vómitos, que no cedían a sintomáticos y que la conduce al servicio de emergencia. Paciente ingresa por el servicio de cirugía general; durante el examen clínico la paciente se encuentra con funciones vitales estables, afebril, despierta, lúcida, orientada en tiempo espacio y persona, quejumbrosa; se evidencia tinte ictérico de mucosas y escleras, no hay alteración en aparato respiratorio y se ausculta un soplo sistólico en foco pulmonar y tricúspideos de intensidad III/ IV; en abdomen se evidencia dolor a la palpación en epigastrio, ruidos hidroaéreos disminuidos y signo de Murphy negativo. El resto del examen físico no fue contributorio.

Con los antecedentes conocidos y la clínica definida se plantea el diagnóstico de pancreatitis aguda biliar, se solicitan exámenes auxiliares para confirmar diagnóstico y se medica con analgésicos y antiespasmódicos.

El hemograma mostró 16,480 leucocitos con $87 \%$ de neutrofilos ; Hemoglobina de $13,6 \mathrm{gr} / \mathrm{dL}$ y plaquetas en 276000 por $\mu \mathrm{L}$. Amilasa de 202 U/L ( VN 0 - 125 ) y Lipasa de 513 U/I ( VN 0 - 78 ). El perfil hepático mostró TGO de 752 U/L; TGP de $508 \mathrm{U} / \mathrm{L}$; Bilirrubina total de $4,2 \mathrm{mg} / \mathrm{dl}$ ( Bilirrubina directa $3,0 \mathrm{mg} / \mathrm{dL}$ ); Fosfatasa alcalina de $191 \mathrm{U} / \mathrm{Lv}(\mathrm{V}<\mathrm{300})$ y Gama glutamil transpeptidasa de $327 \mathrm{U} / \mathrm{L}(\mathrm{VN}<30)$.

La radiografía de tórax (figura 1) mostró una prominencia del cono de la pulmonar sugerente de hipertensión pulmonar. La ecografía abdominal mostró hígado de tamaño y forma habitual, de parénquima con ecogenicidad conservada, con leve dilatación de vías biliares extrahepaticas; colédoco $8 \mathrm{~mm}$. Vesícula biliar en posición no habitual, en la proyección del segmento $\mathrm{VI}$ que mide $46 \times 20 \mathrm{~mm}$ de diámetro, de paredes delgadas y con múltiples imágenes litiasicas en su interior que proyectan sombra acústica posterior. Páncreas de tamaño normal de bordes irregulares y ecotextura ligeramente heterogénea, cabeza 18mm cuerpo $8 \mathrm{~mm}$. Colangioresonancia (figuras 2 a 4): Vesícula biliar de $7 \times 22 \mathrm{~cm}$, de pared delgada ocupada por múltiples cálculos menores de $10 \mathrm{~mm}$. Vesícula biliar y el hilio hepático desplazados a nivel posterior e inferior del hígado. El conducto cístico es largo. Las vías biliares intrahepaticas, el conducto hepático común y los conductos hepático derechos presentan dilatación, colédoco dilatado $(14 \mathrm{~mm}$ de diámetro a nivel del hilio y $6 \mathrm{~mm}$ intrapancreatico) sin evidencia 
de cálculos. El colédoco termina en forma fusiforme en la segunda porción del duodeno. Páncreas de aspecto normal. Ecocardiografía transtorácica Comunicación interauricular con shunt de izquierda a derecha. Función sistólica del ventrículo izquierdo conservada. Crecimiento moderado de cavidades derechas. Hipertensión pulmonar moderada PSAP $50 \mathrm{~mm}$ hg.

Con los hallazgos encontrados la paciente fue diagnosticada de Pancreatitis aguda biliar recurrente asociado a vesícula biliar de localización anormal y anomalía de árbol biliar, así como comunicación interauricular con probable hipertensión pulmonar moderada. La paciente evolucionó favorablemente con remisión del cuadro clínico y normalización de las cifras de amilasa, lipasa y del perfil hepático, siendo trasferida a cirugía para colecistectomía.

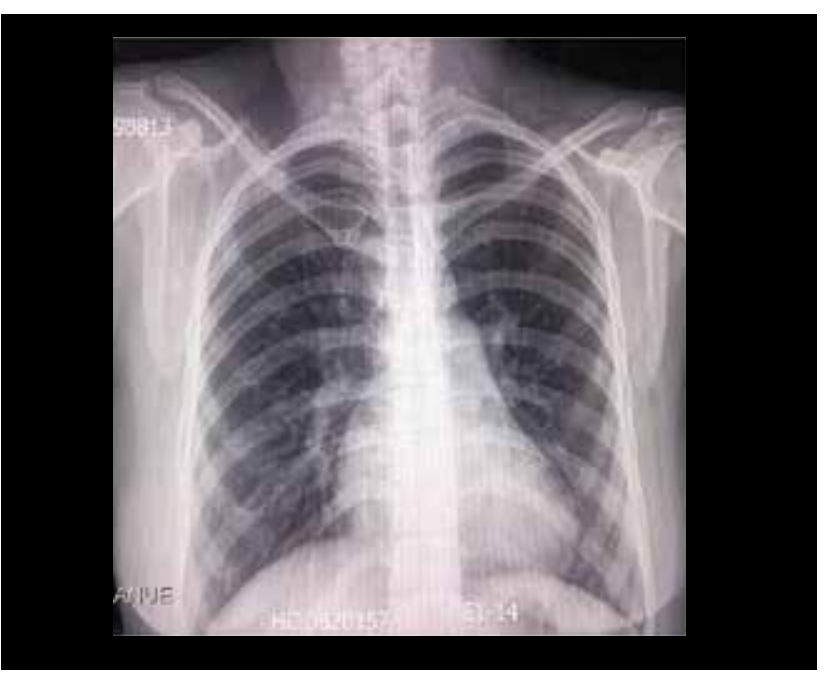

Figura 1. Radiografía de tórax mostrando la presencia de prominencia del cono de la pulmonar asociada a la presencia de comunicación interauricular.

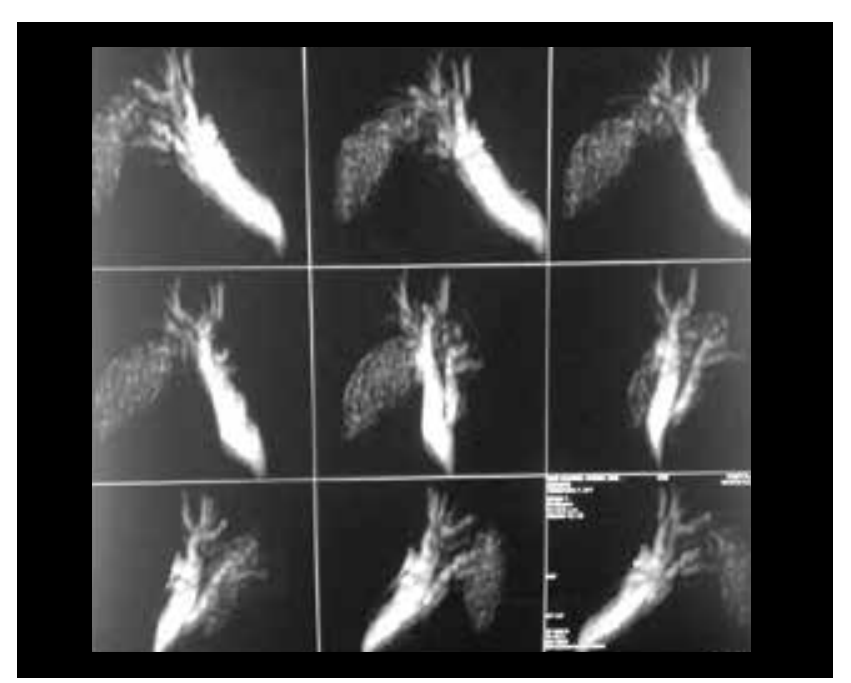

Figura 2. Colangiorresonancia mostrando la presencia de vesícula biliar ocupada totalmente por litos asociada a dilatación de vías biliares intrahepáticas y colédoco.

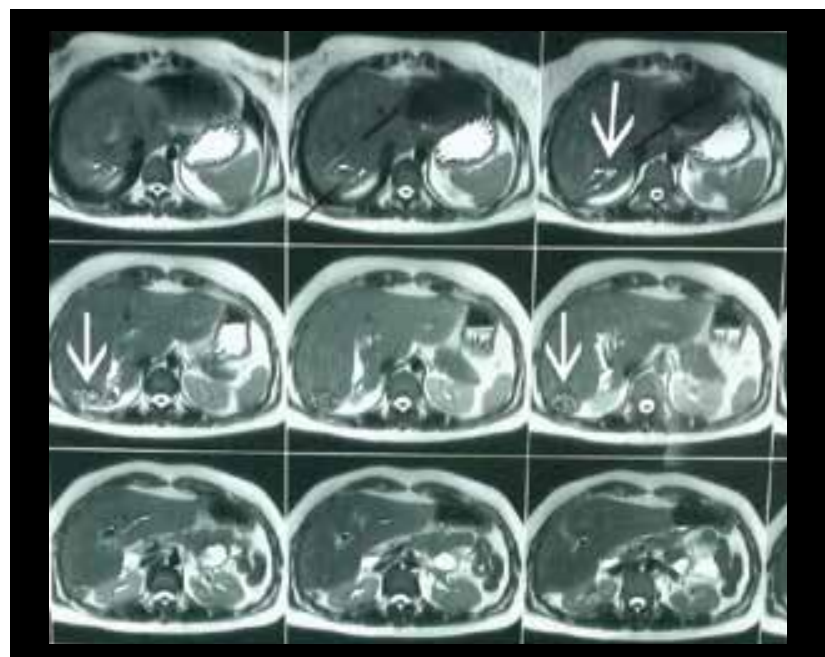

Figura 3. Imagen axial de colangiorresonancia mostrando la localización posterior de la vesícula biliar (flechas).

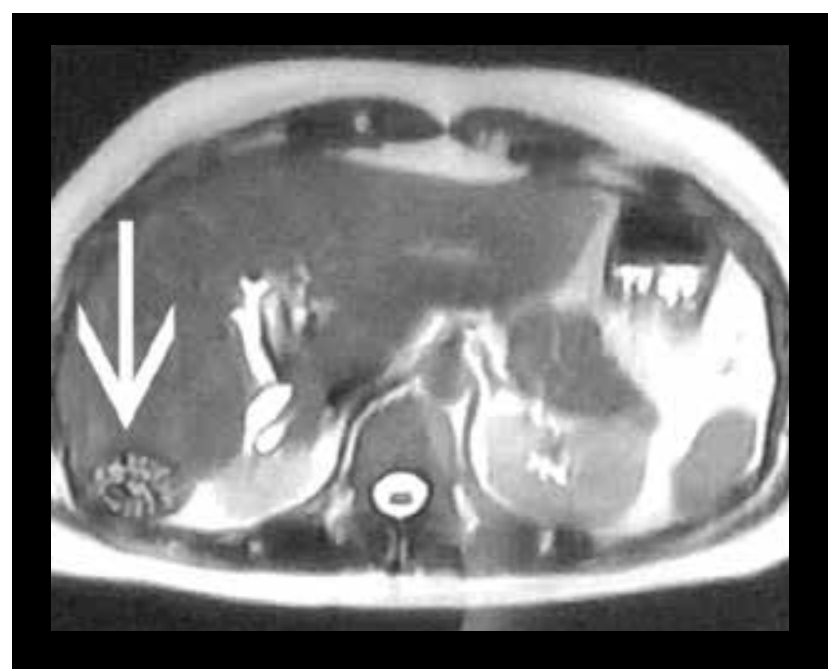

Figura 4. Imagen axial de resonancia magnética a mayor detalle mostrando la presencia de vesícula biliar desplazada posteriormente.

\section{DISCUSIÓN}

La pancreatitis recurrente se define como la presencia de 2 o más episodios de pancreatitis aguda ${ }^{13}$ con resolución clínica entre ambos eventos y puede constituir cerca del $30 \%$ de casos de pancreatitis aguda $^{4}$. Las causas más frecuentes de pancreatitis aguda recurrente son el consumo de alcohol y a la colelitiasis. En nuestra paciente se encontró una vesícula de localización anómala en la región postero inferior hepática con un conducto cístico largo y dilatación de la vía biliar sin presencia de cálculos en su interior la cual cedió luego de algunos días, por lo que podemos suponer que existió una migración de una litiasis coledociana o que se trató de una Odditis asociada a la pancreatitis, esta última condición es también asociada a casos de pancreatitis aguda aún en ausencia de cálculos ${ }^{14}$ 
Entre las anormalidades más frecuentemente reconocidas de la vesícula biliar se encuentra la localización ectópica cuya localización más frecuente es intrahepática, sobretodo en el lóbulo derecho ${ }^{15}$. Las anomalías congénitas de la vesícula biliar pueden clasificarse en alteraciones de localización, número o del desarrollo. La localización ectópica tiene una incidencia de 0,1-0,7\% siendo con mayor frecuencia intrahepática, en el lóbulo derecho ${ }^{15}$. Es una anomalía del desarrollo que impide que la vesícula biliar se mueva desde su posición intrahepática en el segundo mes de gestación hasta su localización superficial normal ${ }^{16}$. Presenta una función alterada porque no se vacía completamente lo que puede resultar en la formación de cálculos biliares debido al estasis ${ }^{17}$. La colecistectomía laparoscópica es la técnica quirúrgica de elección en la vesícula biliar intrahepática ${ }^{18}$. La vesícula biliar aberrante o en lugar no habitual, aunque muchas veces se descubre con la ultrasonografía y la tomografía axial computarizada (TAC), en otras se encuentra durante el acto quirúrgico. En el caso discutido consideramos que la presencia en una localización anatómica anormal en conjunto con un cístico alargado son factores que pueden haber contribuido a la estasis biliar y la consiguiente formación de cálculos.

En los casos de pancreatitis recurrente se debe garantizar una adecuada historia clínica registrando el consumo de tabaco, alcohol, fármacos, antecedentes de vasculitis, infecciones, trauma e historia familiar de pancreatitis. La anamnesis adecuada, junto con un examen físico apropiado son esenciales para evaluar la etiología de la pancreatitis aguda recurrente. Los estudios bioquímicos deben incluir calcio, triglicéridos, amilasa, lipasa y perfil hepático. La ecografía abdominal está indicada para detectar litiasis biliar. Se recomienda repetirla después del episodio inicial si la evaluación ecográfica ha estado limitada por la presencia de íleo o dolor, particularmente en ausencia de patología biliar evidente. La Tomografía abdominal es útil para detectar alteraciones morfológicas del páncreas tales como el páncreas divisum así como, la extensión y gravedad de la pancreatitis, dilatación del Wirsung y la presencia de neoplasia. Cabe mencionar que en un $7 \%$ de los pacientes con pancreatitis recurrente coexisten varias etiologías.

La paciente presentó además como hallazgo incidental la presencia de una comunicación interauricular asociada a hallazgos ecocardiográficos sugerentes de hipertensión pulmonar moderada. Aunque se ha reportado la coexistencia de comunicación interauricular con agenesia de vesícula biliar19,20, no hemos encontrado en la literatura otros reportes sobre la coexistencia con anomalías en la localización de la vesícula biliar.

Contribuciones de autoría: Los autores participaron en la generación, recolección de información, redacción y versión final del artículo original.

Financiamiento: Autofinanciado.

Conflicto de interés: Los autores declaran no tener conflictos de interés en la publicación de este artículo.

Recibido: 03 de febrero 2019

Aprobado: 05 de mayo 2019

Correspondencia: Alonso Soto

Dirección: Av. Alfredo Benavides 5440, Santiago de Surco 15039, Lima-Perú. Teléfono: (01) 7080000

Correo: sotosolari@gmail.com

\section{REFERENCIAS BIBLIOGRÁFICAS}

1. Steinberg WM, Chari ST, Forsmark CE, Sherman S, Reber HA, Bradley EL 3rd, DiMagno E. Controversies in clinical pancreatology: management of acute idiopathic recurrent pancreatitis. Pancreas 2003; 27: 103-117.

2. Sajith KG, Chacko A, Dutta AK. Recurrent Acute Pancreatitis: Clinical Profile and an Approach to Diagnosis. Dig Dis Sci 2010; 55:3610-3616.

3. Guda NM, Romagnuolo J, Freeman ML. Recurrent and relapsing pancreatitis. Curr Gastroenterol Rep 2011; 13:140-149.

4. Gullo L, Migliori M, Pezzilli R, Olah A, Farkas G, Levy P et al. An update on recurrent acute pancreatitis: data from five European countries. Am J Gastroenterol 2002; 97: 1959-1962.

5. Lankisch PG, Breuer N, Bruns A et al. Natural history of acute pancreatitis: a long term population-based study. Am J Gastroenterol 2009; 104:2797-2805.

6. Joergensen M, Brusgaard K, Cruger DG, et al.: Incidence, prevalence, etiology, and prognosis of first-time chronic pancreatitis in young patients: a nationwide cohort study. Dig Dis Sci 2010, 55:2988-2998.

7. Patel RS, Johlin FC, Murray JA. Celiac disease and recurrent pancreatitis. Gastrointest Endos. 1999;50(6):823-7.
8. Shimosegawa T, Chari ST, Frulloni L et al. International Consensus Diagnostic Criteria for Autoimmune Pancreatitis. Guidelines of the International Association of Pancreatology. Pancreas 2011;40:352-358.

9. Testoni PA, Mariani A, Curioni S, Zanello A, Masci E. MRCP-secretin testguided management of idiopathic recurrent pancreatitis: long-term outcomes. Gastrointest Endosc 2008;67:1028-34

10. Jorba R, Fabregat J, Borobia FG, Busquets J, Ramos E, Torras J et al. Neoplasias quísticas del páncreas. Manejo diagnóstico y terapéutico. Cir Esp 2008; 84(6):296-306.

11. Venkatesh PGK, Navaneethan U, Vege SS. Intraductal Papillary Mucinous Neoplasm and Acute Pancreatitis. J Clin Gastroenterol 2011;45:755-758.

12. Hamlin JA. Anomalías del árbol biliar. En: Berk JE, Haubrich WS, Kalser MH, Roth JLA, Schaffner F. Bockus-Gastrenterología. Ed. Salvat. 4. ed. Barcelona, España 1987: 3801-3814

13. Testoni PA. Acute recurrent pancreatitis: Etiopathogenesis,diagnosis and treatment. World J Gastroenterol 2014; 20(45):16891-16901. Disponible en http://www.wjgnet.com/1007-9327/full/v20/i45/16891.htm 
14. Elta GH. Sphincter of Oddi dysfunction and bile duct microlithiasis in acute idiopathic pancreatitis. World J Gastroenterol 2008; 14(7): 1023-1026. Disponible en http://www.wjgnet.com/1007-9327/14/1023.asp

15. Guiteau JJ, Fisher M, Cotton RT, Goss JA. Intrahepatic Gallbladder. J Am Coll Surg. 2009;209:672.

16. Audi $P$, Noronha F, Rodrigues J. Intrahepatic gallbladder A case report and review of literature. The Internet Journal of Surgery 2009;24:133-5.

17. Wysong Ch, Gorten RJ. Intrahepatic Gallbladder. Southern Medical Journal 1980;73:825-6.
18. Segura JJ, Navarro A, Ashrafian H, Ruescas FJ, Cuiñas K, Martínez-Isla A. Abordaje Laparoscópico de la Vesícula Biliar Intrahepática. Cir Esp. 2014;92(Espec Congr):514.

19. LangerT, Baudenbacher R, HablützelK, Kehl O. Die Gallenblasenagenesie: ein diagnostisches Problem? Schweiz Rundsch Med Prax. 1989 May 30;78(22):646-8.

20. Liu Y, Ao Nl, Zhao H. Gallbladder agenesis and atrial septal defect: A case report. Exp Ther Med. 2016 May;11(5):1795-1798. Epub 2016 Feb 29.

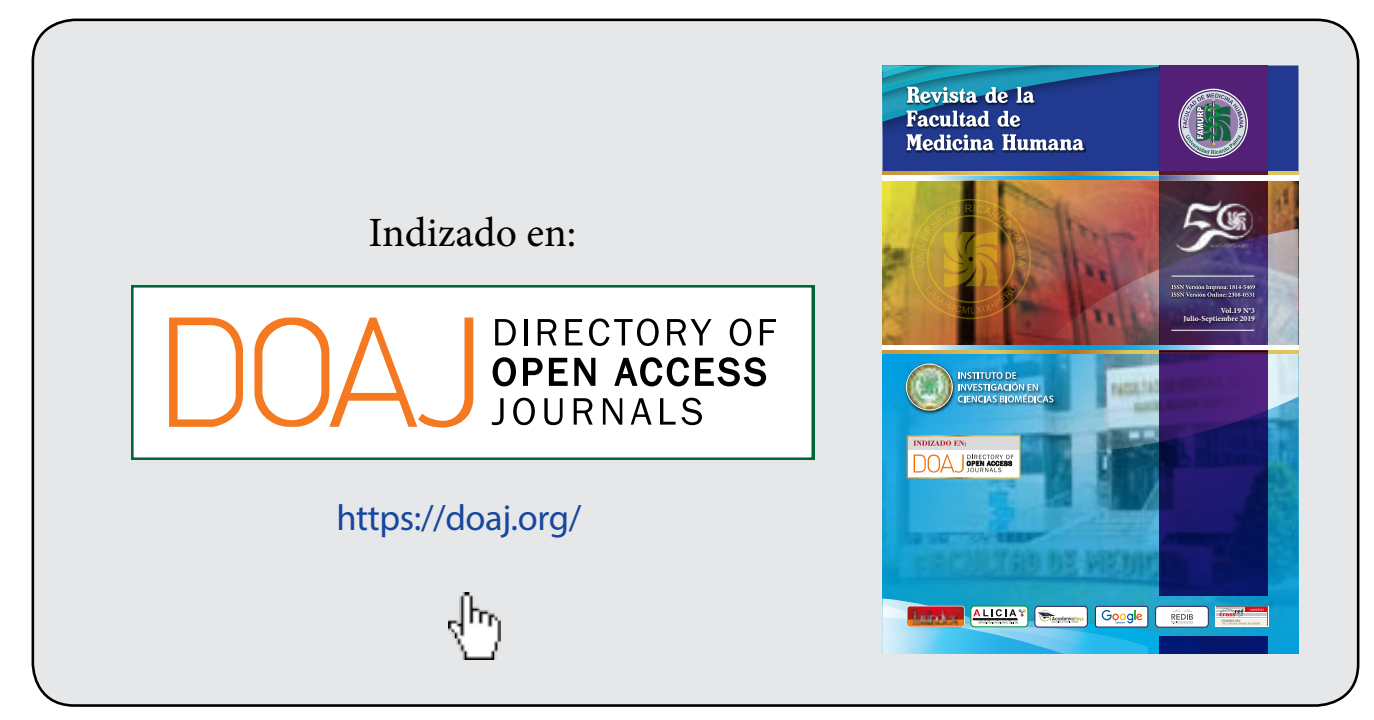

